\title{
A nonextensive method for spectroscopic data analysis with artificial neural networks
}

\author{
Dimitrios Kalamatianos \\ Hamilton Institute, National University of Ireland, Maynooth, Ireland
}

Aristoklis D. Anastasiadis*

Electrical and Computer Engineering Department, University of Patras, Rio, Achaia 26500, Greece and Centro Brasileiro de Pesquisas Fisicas, Rua Xavier Sigaud 150 22290-180 Rio de Janeiro Brazil

Panos Liatsis

School of Engineering and Mathematical Sciences, City University, Northampton Square, London EC1V OHB, UK

(Received on 16 January, 2009)

\begin{abstract}
In this paper we apply an evolving stochastic method to construct simple and effective Artificial Neural Networks, based on the theory of Tsallis statistical mechanics. Our aim is to establish an automatic process for building a smaller network with high classification performance. We aim to assess the utility of the method based on statistical mechanics for the estimation of transparent coating material on security papers and cholesterol levels in blood samples. Our experimental study verifies that there are indeed improvements in the overall performance in terms of classification success and at the size of network compared to other efficient backpropagation learning methods.
\end{abstract}

Keywords: Nonextensive statistical mechanics, Neural networks, Pattern classification, Spectroscopy

\section{INTRODUCTION}

Neural networks are very sophisticated modeling techniques capable of modeling extremely complex functions. Nowadays, they are being successfully applied across a wide range of problem domains, in areas such as finance, medicine, engineering, geology and physics [1-3]. Indeed, anywhere that there are problems of prediction or classification, neural networks are being introduced. Therefore, Artificial Neural Networks (ANNs) are well suited for both pattern recognition, classification or clustering and quantitative modeling.

One example of quantitative modeling application is the classification of transparent coating material on papers. Coated paper finds its application in a vast variety of industrial needs. The role of coating consists in the creation of smooth surface for good print ability and high surface gloss. Artificial Neural Networks have been applied to solve this type of classification problem. Specifically ANNs to the quantitative analysis of paper coatings using infrared spectra have been reported before and proved to be reliable and effective analysis tools [4]. Kohonen self-organizing networks [4] are one of the most prominent tools for unsupervised learning and Feed-forward neural networks with learning algorithms mainly grown from error backpropagation [5] are extremely useful for building complex relationships between inputs and outputs sets of parameters. The experience in applying artificial neural networks in these types of problems is very extensive. However, neural network error surfaces are characterized by a number of unhelpful features, such as local minima, flat-spots and plateaus, saddle-points, and long narrow ravines making the efficient training of the ANNs difficult sometimes. Many training algorithms have been proposed so far to improve neural network performance [6-8].

A variety of approaches inspired from the unconstrained

${ }^{*}$ Corresponding Author: anastasiadis@upatras.gr optimisation theory have also been applied, in order to use second derivative related information to accelerate the learning process $[9,10]$. Nevertheless, it is not certain that the extra computational cost these methods require leads to speedups of the minimisation process for non-convex functions when far from a minimiser [11]. This problem can be overcome through the use of global optimisation. However, the drawback of this class of methods is that they are very computationally expensive. Statistical mechanical methods have also been successfully applied to the study of neural network models of associative memory [12]. Another class of efficient training algorithms is the conjugate gradient learning based schemes. These methods have a second-order convergence property without complex calculation of the Hessian matrix.

In this work, emphasis will be given on the construction and training of feed-forward neural networks using gradient based algorithms by applying a nonextensive formula $[2,13-$ $15]$ in the error expression. We compare this class of methods with gradient descent error minimization based algorithms, which are widely used with success in such classification problems. We also present experimental results from two problems in spectroscopy. We use feedforward neural networks to estimate transparent coating material on security papers and serum cholesterol levels in blood. In both problems we apply an evolving stochastic learning algorithm for neural networks based on the theory of Tsallis statistical mechanics $[14,16]$. Our approach combines a quick and computationally cheap local search method with a global search technique inspired in the generalized entropy of nonextensive statistical mechanics, and replaces the usual Boltzmann-Gibbs factor used in simulated annealing by the $q$ - exponential function[13, 14]. This global search technique possess strong convergence properties, and, at least in principle, are straightforward to implement and apply. The experimental study verifies that the synergy of techniques from nonextensive statistics provides neural learning schemes with small size structure and high classification performance. In particular, results show that the prediction of coat weights is possible with an 
error in the order of $10^{-3}$ while cholesterol prediction is a much more difficult problem. However, in both cases, the error can be minimized by applying a nonextensive formula in the error function. We also show that this method can reduce the ANN's architecture for the construction of more suitable mathematical models and at the same time we can achieve a noteworthy overall classification performance.

The organization of the paper is as follows. In Section 2 we explain some of the aspects of Nonextesive Statistical Mechanics applied in Neural Networks. Section 3 gives the characteristics of the proposed nonextensive method and explains some aspects of the training algorithms that are important for the problem at hand. Next section describes the available data and experimental methods followed by the results and discussion. Finally, we draw the conclusions and suggestions for future investigations.

\section{NONEXTENSIVE STATISTICAL MECHANICS}

Statistical mechanics set out to explain the behavior of macroscopic systems by studying the statistical properties of their microscopic constituents [17].

Nowadays the idea of nonextensivity has been used in many applications. Nonextensive statistical mechanics [14] have successfully been applied in physics (astrophysics, astronomy, cosmology, nonlinear dynamics etc) [18, 19], chemistry [3], biology [20], human sciences [21], economics [22], computer sciences [2, 23, 24], and others [25].

Nonextensive statistical mechanics are based on Tsallis entropy. Tsallis statistics are currently considered useful in describing the thermostatistical properties of nonextensive systems; it is based on the generalized entropic form [14]:

$$
S_{q} \equiv k \frac{1-\sum_{i=1}^{W} p_{i}^{q}}{q-1} \quad(q \in \Re)
$$

where $W$ is the total number of microscopic configurations, whose probabilities are $\left\{p_{i}\right\}$, and $k$ is a conventional positive constant. When $q=1$ it reproduces the Boltzmann-Gibbs (BG) entropic form $S_{B G}=-k \sum_{i=1}^{W} p_{i} \ln p_{i}$. The nonextensive entropy $S_{q}$ achieves its extreme value at the equiprobability $p_{i}=1 / W, \forall i$, and this value equals $S_{q}=k \frac{W^{1-q}-1}{1-q}$ $\left(S_{1}=S_{B G}=k \ln W\right)[14,25]$. The Tsallis entropy is nonadditive in such a way that, for statistical independent systems $A$ and $B$, the entropy satisfies the following property:

$$
\frac{S_{q}(A+B)}{k}=\frac{S_{q}(A)}{k}+\frac{S_{q}(B)}{k}+(1-q) \frac{S_{q}(A)}{k} \frac{S_{q}(B)}{k} .
$$

It is subadditive for $q>1$, superadditive for $q<1$, and, for $q=1$, it recovers the $\mathrm{BG}$ entropy, which is additive [25]. The Boltzmann factor is generalized into a power-law. The mathematical basis for Tsallis statistics includes $q$-generalized expressions for the logarithm and the exponential functions, which are the $q$-logarithm and the $q$-exponential functions. The $q$-exponential function, which reduces to $\exp (x)$ in the limit $q \rightarrow 1$, is defined as follows

$$
e_{q}^{x} \equiv[1+(1-q) x]^{\frac{1}{(1-q)}}=\frac{1}{[1-(q-1) x]^{\frac{1}{(q-1)}}}\left(e_{1}^{x}=e^{x}\right)
$$

We remind that extremizing entropy $S_{q}$ under appropriate constraints we obtain a probability distribution, which is proportional to q-exponential function.

In the following sections we discuss how we could obtain successful results by applying the nonextensive entropy in training feedforward neural networks. The next section introduces an adaptive search strategy that aims to alleviate the problem of occasional convergence to local minima in supervised training, achieving high classification performance in coat weight estimation and cholesterol problems via a simple structure of feedforward neural network.

\section{THE PROPOSED MODEL}

In this work we focus on gradient descent based algorithms for supervised learning of neural networks. The most popular training algorithm of this category is the batch BackPropagation (BP) [26]. It is a first order method that minimizes the error function by updating the weights using the steepest descent method [9]:

$$
w(t+1)=w(t)-\eta \nabla E(w(t))
$$

where $E$ is the batch error measure defined as the Sum of Squared differences Error function (SSE) over the entire training set, and $t$ indicates iterations (time). The $\nabla(E)$ is the gradient vector, which is computed by applying the chain rule on the layers of the FNN[26]. The parameter $\eta$ is a heuristic, called learning rate. The proper value of $\eta$ depends on the shape of the error function. The learning rate values help to avoid convergence to a saddle point or a maximum. In order to secure the convergence of the BP training algorithm and avoid oscillations in a steep direction of the error surface, a small learning rate is chosen $(0<\eta<1)$.

Our approach adapts the weights using only information from the sign of a gradient vector, which is calculated on a perturbed error function, and uses adaptive steps along each weight directions. The perturbations are generated from a noise sources that replaces the usual Boltzmann-Gibbs factor used in annealing schedules by the q-exponential function of the generalised entropy of nonextensive statistical mechanics [14, 27].

Following the above discussion and inspired by [13], in our method, noise is generated according to a schedule that can be expressed as:

$$
Q(T, k)=e_{q}^{-T(\ln 2) \cdot k}=[1-(1-q) T(\ln 2) \cdot k]^{\frac{1}{1-q}},
$$

where $T$ is the temperature; $k$ indicates iterations. Noise is not applied proportionally to the size of each weight; instead, a form of weight decay is used, which is considered beneficial for achieving a robust neural network that generalizes well. Thus, noise is introduced by formulating the perturbed error function:

$$
\tilde{E}\left(w^{k}\right)=E\left(w^{k}\right)+\mu \cdot \sum_{i=1}^{n} \frac{\left(w_{i}^{k}\right)^{2}}{\left[1+\left(w_{i}^{k}\right)^{2}\right]} \cdot Q(T, k),
$$

where $E(w)$ is the batch error function, $\sum_{i} w_{i}^{2} /\left(1+w_{i}^{2}\right)$ is the weight decay bias term which can decay small weights more 
rapidly than large weights, and $\mu$ is a parameter that regulates the influence of the combined weight decay/noise effect. This form of weight decay modifies the error landscape so that smaller weights are favored at the beginning of the training but as learning progresses the magnitude of the weight decay is reduced to favor the growth of large weights. Thus, as the error landscape is modified during training, the search method is allowed to explore regions of the error surface that were previously unavailable. Minimization of (6) requires calculating the gradient of the error function with respect to each weight

$$
\tilde{g}_{i}\left(w^{k}\right)=g_{i}\left(w^{k}\right)+\mu^{\prime} \cdot \frac{w_{i}^{k}}{\left[1+\left(w_{i}^{k}\right)^{2}\right]^{2}} \cdot Q(T, k),
$$

where $\mu^{\prime}>0$.

The proposed Hybrid Training Scheme (HTS) applies a sign-based weight adjustment, on the perturbed error function (Eq. 6) using the gradient term of Eq. (7). The direction of the weights are changed by the following procedure:

$$
w^{k+1}=w^{k}-\operatorname{diag}\left\{\eta_{1}^{k}, \ldots, \eta_{i}^{k}, \ldots, \eta_{n}^{k}\right\} \operatorname{sign}\left(\tilde{g}_{i}\left(w^{k}\right)\right),
$$

where $k$ indicates iterations; $\operatorname{diag}\left\{\eta_{1}, \ldots, \eta_{n}\right\}$ defines the $n \times n$ diagonal matrix with elements $\eta_{1}, \ldots, \eta_{n}$, and $\eta_{i}^{k}(i=$ $1,2, \ldots, n)$ are the $k$-th iteration stepsizes that receive small positive real values, also called learning rates as their role is to control the amount of weight adjustments and thus directly to affect the rate of the learning process. The $\operatorname{sign}\left(\tilde{g}_{i}\left(w^{k}\right)\right)$ denotes the column vector of the signs of the components of $\tilde{g}_{i}\left(w^{k}\right) ; \tilde{g}(w)^{\top}=\left(\tilde{g}_{1}(w), \ldots, \tilde{g}_{n}(w)\right)$ defines the transpose of the gradient $\nabla E(w)$ of the sum-of-squared-differences error function $E$ at $w$; if the sign of the gradient of the perturbed error function (7) has remained the same then $\eta_{i}^{k}$ is calculated by the following equation:

$$
\begin{aligned}
& \text { if } \quad\left(\tilde{g}_{i}\left(w^{k-1}\right) \cdot \tilde{g}_{i}\left(w^{k}\right)>0\right) \quad \text { then } \\
& \eta_{i}^{k}=\min \left(\eta_{i}^{k-1} \cdot \eta^{+}, \Delta_{\max }\right)
\end{aligned}
$$

where $0<\eta^{-}<1<\eta^{+}, \Delta_{\max }$ is the stepsize upper bound. When the gradient is zero then the update value is multiplied by the learning rate $\eta_{i}^{k}$ which remains the same.

Finally, if only the sign of the gradient has changed, then the following rule is used:

$$
\begin{aligned}
& \text { if } \quad\left(\tilde{g}_{i}\left(w^{k-1}\right) \cdot \tilde{g}_{i}\left(w^{k}\right)<0\right) \text { then } \\
& \eta_{i}^{k}=\max \left(\eta_{i}^{k-1} \cdot \eta^{-}, \Delta_{\text {min }}\right)
\end{aligned}
$$

The Adaptive Hybrid Training Scheme (AHTS) works by adapting the learning rates following a rational similar to HTS algorithm by applying a cooling temperature procedure. This defines the relationship between $T$ and $q$ values. The application of cooling helps to regulate the training algorithm, making it more deterministic. This new Adaptive Hybrid training Scheme-AHTS behaves in a more stochastic way, during the initial stages, and then becomes more deterministic as the number of iterations increases. Thus, when we are close to the minimizer, the algorithm hopefully will avoid oscillations and converge faster. The cooling procedure is described by the next equation:

$$
T=T_{0} \cdot\left[\frac{2^{q-1}-1}{(1+k)^{q-1}-1}\right], q>1
$$

where $T_{0}$ is the initial temperature, $T$ is the current temperature, $k$ is the number of iterations, and $q$ is the Tsallis entropic index.

The challenge is to cool the temperature as quick as we can, but still having the ability to converge to global minimum with high probability.

We have followed the recommendations of [2] in setting the parameters: (i) the increase factor is set to $\eta^{+}=1.2$; (ii) the decrease factor is set to $\eta^{-}=0.5$; (iii) the initial stepsize for all $i$ is set to $\eta^{0}=0.1$; (iv) the maximum allowed stepsize, which is used in order to prevent the weights from becoming too large, is $\Delta_{\max }=50 ;(\mathrm{v})$ the minimum allowed stepsize $\Delta_{\min }=10^{-6}$.

\section{EXPERIMENTAL PROCEDURES}

Two data sets were used for this study, both from the area of spectroscopy. In the first case, spectra were collected with a novel Fourier transform Michelson interferometer [28] and operating in the near-infrared (NIR) range. The paper samples provided for these experiments were security papers with added coating to improve printing and aesthetic quality of documents. Five sheets were supplied with coat weights of $3.3,4.2,5.2,6.2$ and $6.9 \mathrm{~g} / \mathrm{m}^{2}$ respectively. Each paper sample was supplied as an A4-sized sheet, which was subdivided into six equally sized samples, i.e., from each of the original five sheets, six smaller samples were obtained, each having approximate dimensions of $10.5 \times 9.87 \mathrm{~cm}$. The six samples from each sheet were numbered 1 to 6 . Thus, the interferograms from different parts of a particular sample could be compared directly.

Spectral information was collected as 3000 (100 from each of $5 \times 6=30$ samples) double-sided interferograms with 1024 points. The interferograms were phase compensated and were, therefore, symmetrical about the centreburst. Consequently, it was possible to discard half of the data without incurring any loss of information and the dimensionality of the feature space was reduced from 1024 to 512. The first 256 points of the mean interferograms from each coated paper are shown in Fig. 1.

The 3000 interferograms from the coated sides of the paper samples were divided into training, $\mathbf{X}$, validation, $\mathbf{Z}$, and testing, $\mathbf{Y}$, sets with dimensionality $512 \times 1800,512 \times 600$ and $512 \times 600$, respectively ( $60 \%$ for training, $20 \%$ for validation and $20 \%$ for testing). A series of experiments was undertaken to train multi-layer perceptron (MLP) neural networks [29] of various sizes. As can be seen in Fig. 1, there is little variation and hence discriminating information content in the interferograms beyond the 20th data point from the centreburst. In addition, the autocorrelation sequence of the average interferogram was calculated and it was observed that it drops significantly after the first 16 lags. Thus, the number of inputs, i.e., data points presented to the network was incremented from 1 to 20 , and for each increment, an MLP was 


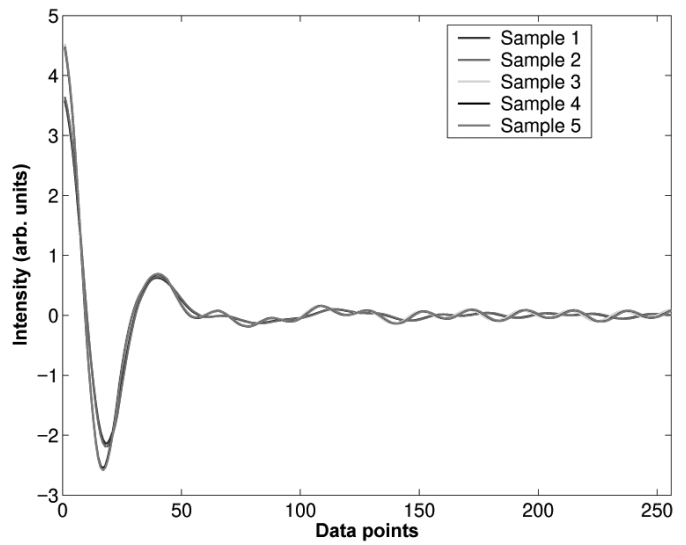

FIG. 1: Plot of the mean interferograms from each coated paper obtained from the Michelson prototype instrument. The first 256 points to the right of the centreburst are shown.

simulated in Matlab with one hidden layer, whose size was systematically varied between 1 and 30 nodes. Experiments showed that further increase of the number of hidden nodes did not improve the generalization due to overfitting. For hidden layer nodes, hyperbolic tangent transfer functions were used, whereas logistic functions were used for output layer nodes. Ten trials were attempted for each network and the MSE of prediction with the number of epochs required for convergence were recorded. The criterion for stopping training was an $\mathrm{MSE} \leq 10^{-5}$ or an increment of the validation error for a specified number of iterations.

The second data set used was from a medical application [30] and contained a total of 264 patients for which measurements of 21 wavelengths of the spectrum has been collected. For the same patients we also have measurements of HDL, LDL, and VLDL cholesterol levels, based on serum separation. The first step was to perform a principal component analysis and retain those principal components which account for $99.9 \%$ of the variation in the data. There is significant redundancy in the data set, since the principal component analysis has reduced the size of the input vectors from 21 to 4 . The next step is to divide the data up into training, validation and test subsets. We will take $25 \%$ of the data for the validation set, $25 \%$ for the test set and $50 \%$ for the training set. We pick the sets as equally spaced points throughout the original data.

A similar procedure as described above for the paper data was used, but this time the number of inputs to the network was fixed to 4 principal components, and an MLP was simulated in Matlab with one hidden layer, whose size was systematically varied between 1 and 30 nodes. For hidden layer nodes, hyperbolic tangent transfer functions were used, whereas linear transfer functions were used for output layer nodes. Ten trials were attempted for each network and the stopping criterion was the same as above.

We compared our proposed model to different gradient descent algorithms which use constant or adaptive learning rates as these methods show improved learning speed and good convergence behavior [8]. More specifically, we first applied the batch gradient descent training algorithm (TRAINGD).

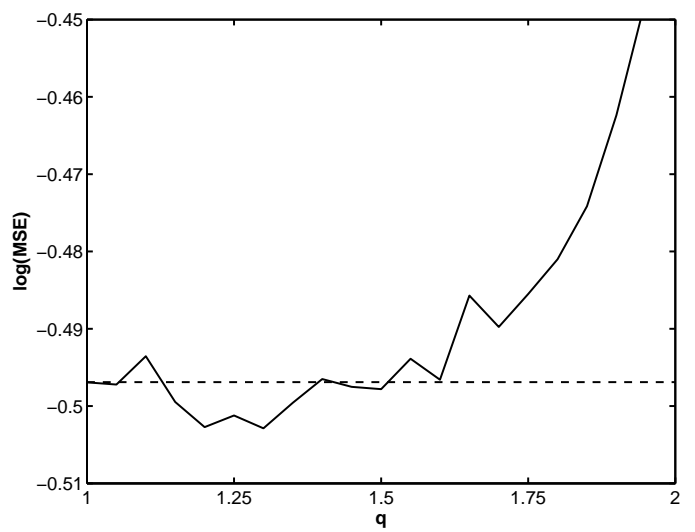

FIG. 2: Mean MSE of networks with 1 to 30 nodes for $1<q<2$.

Next we investigated the performance of the gradient descent algorithm with momentum (TRAINGDM). We also applied gradient descent with adaptive learning rate with (TRAINGDX) or without momentum (TRAINGDA). Nevertheless, by applying all these algorithms there is no guarantee that the network error will monotonically decrease at each iteration and that the weight sequence will converge to a minimizer of the sum-of-squared-differences error function $E$. Therefore, as shown in the next section, the proposed nonextensive training schemes provide evidence that there is significantly improvement in the overall classification performance.

\section{RESULTS AND DISCUSSION}

To find the optimal values of $q$, a series of runs were performed using the cholesterol data as input and changing the network's structure. For each $0<q<5$, the mean MSE of all networks (from 1 to 30 neurons) was recorded and it was found that there is an optimal area between $q=1.2$ and $q=1.3$. Figure 2 shows the results of the tests around that area in logarithmic scale. The minimum value of MSE is 0.3141 and is achieved for $q=1.3$. It is also shown that the error for $q$ between 1.15 and 1.5 is lower than the error obtained with $q=1$.

As mentioned in the previous section, in the case of cholesterol data the number of inputs was kept constant and the number of nodes was varied from 1 to 30 . Using the optimal value of $q$ calculated above $(q=1.3)$ the lowest prediction error was found to be 0.22 and it was obtained with a network with 25 nodes. A summary of the results can be seen in Table I. The first column shows the network structure that gave the minimum error and the last column shows the mean MSE recorded for reduced size networks with a maximum of 15 neurons. The error is relatively high but this is considered to be a difficult classification problem. Putting the entire data set through the network (training, validation and test) and performing a linear regression between the network outputs and the corresponding targets we can get a better idea of the network response. The results are shown in Figs. 3 and 4. Fig. 3 shows the results obtained with the network structure that gave the smallest prediction error trained with the HTS. 
TABLE I: Optimal topologies and MSE for MLP networks trained with spectral components of blood samples.

\begin{tabular}{|c|c|c|c|c|}
\hline $\begin{array}{c}\text { Training } \\
\text { Method }\end{array}$ & $\begin{array}{c}\text { Best } \\
\text { Topology }\end{array}$ & $\begin{array}{c}\text { Best } \\
\text { MSE }\end{array}$ & $\begin{array}{c}\text { Mean } \\
\text { MSE }\end{array}$ & $\begin{array}{c}\text { \%) Improvement } \\
\text { MSE of AHTS }\end{array}$ \\
\hline AHTS & $4-25-3$ & 0.220 & 0.349 & - \\
\hline HTS & $4-27-3$ & $0.245(+)$ & $0.352(-)$ & $0.85 \%$ \\
\hline GD & $4-23-3$ & $0.343(+)$ & $0.507(+)$ & $31.2 \%$ \\
\hline GDX & $4-30-3$ & $0.292(+)$ & $0.422(+)$ & $17.3 \%$ \\
\hline GDM & $4-13-3$ & $0.337(+)$ & $0.529(+)$ & $34.0 \%$ \\
\hline GDA & $4-13-3$ & $0.323(+)$ & $0.421(+)$ & $17.1 \%$ \\
\hline
\end{tabular}

TABLE II: Optimal topologies and MSE for MLP networks trained with spectral components of coated paper data.

\begin{tabular}{|c|c|c|c|c|}
\hline $\begin{array}{c}\text { Training } \\
\text { Method }\end{array}$ & $\begin{array}{c}\text { Best } \\
\text { Topology }\end{array}$ & $\begin{array}{c}\text { Best } \\
\text { MSE }\end{array}$ & $\begin{array}{c}\text { Mean } \\
\text { MSE }\end{array}$ & $\begin{array}{c}\text { \%) Improvement } \\
\text { MSE of AHTS }\end{array}$ \\
\hline AHTS & $2-24-1$ & $3.1 \times 10^{-3}$ & $4.2 \times 10^{-3}$ & - \\
\hline HTS & $17-15-1$ & $3.1 \times 10^{-3}(=)$ & $5 \times 10^{-3}(+)$ & $16 \%$ \\
\hline GD & $7-23-1$ & $4 \times 10^{-3}(+)$ & $7.6 \times 10^{-3}(+)$ & $44.7 \%$ \\
\hline GDX & $7-24-1$ & $3.7 \times 10^{-3}(+)$ & $6.9 \times 10^{-3}(+)$ & $39.1 \%$ \\
\hline GDM & $18-24-1$ & $4 \times 10^{-3}(+)$ & $8.7 \times 10^{-3}(+)$ & $51.7 \%$ \\
\hline GDA & $6-19-1$ & $4 \times 10^{-3}(+)$ & $7.3 \times 10^{-3}(+)$ & $42.5 \%$ \\
\hline
\end{tabular}

Fig. 4 shows the linear regression applied to network outputs when trained with all the schemes in test. The network structures are shown in the first column of Table I. We see that the Hybrid Training Schemes give not only the lowest error but also perform better than the gradient descent algorithms tested for smaller sizes of networks.

Similar results were obtained using paper data as inputs. In this case, the error was significantly reduced compared to the network outputs with the blood samples because this is naturally an easier problem. The lowest prediction error was found to be $3.1 \times 10^{-3}$ and it was obtained with a 2-24-1 network structure. Output results can be seen in Table II.

We have evaluated the performance of the new methods and compared them with the class of the gradient descent algorithms. The statistical significance of the results has been analyzed using the Wilcoxon test [31]. This is a nonparametric method that is considered an alternative to the paired ttest. All statements in the tables reported below refer to a significance level of 0.05 . Statistically significant cases are marked with (+), while (-) shows the cases that do not satisfy the significance level.

Going through the experimental results with the cholesterol data, we can conclude that the first two outputs seem to track the targets reasonably well, and the R-values are almost 0.9. The third output (VLDL levels) is not so well modeled. Improved results may be obtained with Bayesian regularization instead of early stopping for our training technique. Of course there is also the possibility that VLDL levels cannot be accurately computed based on the given spectral components. Nevertheless, HTS and AHTS gave the best overall mean squared error and looking at the slopes of the regression lines of Fig. 4 we can say that they best modelled output 1 , and only TRAINGDX gave slightly better results for outputs 2 and 3 .

In any case, different data pre-processing strategies could be applied to improve network performance. Both the construction requirements of ANN models with correct prediction ability and the poor agreement between the number of spectral points and the number of spectra in small data sets require to find different strategies for the reduction of the size of the input vector. In this paper, though, we have focused on training schemes and tried to relieve the benefits of nonextensive statistical mechanics when applied to feedforward neural networks.

\section{CONCLUSIONS}

The main goal of this study was to test the performance of ANNs with nonextensive learning methods. It has been observed that they can be used as an efficient tool to estimate coat weights on security papers and blood cholesterol levels. Preliminary numerical results using supervised learning schemes through Nonextensive Statistical Analysis, show improved performance in terms of prediction error and network structure compared to the best previous attempts. This is mainly because of the use of more stochastic neural schemes. In particular, the use of Adaptive Hybrid Training Scheme improved the network performance by as much as $34 \%$ for the classification of blood cholesterol levels and $51 \%$ for coating weight estimation. To obtain these results a series of experiments was performed to identify the optimal values of $q$. These experiments also showed that the error was reduced for $q \neq 1$ compared with that obtained for $q=1$. During the study, emphasis was given on the performance of smaller network architecture, thus reducing the computation cost. Training with nonextensive statistical mechanics proved to be particularly beneficial with reduced size networks. Future work will focus on exploring the use of the new method not only in spectroscopy but also in other classes of problems, possibly in combination with more efficient dimensionality reduction techniques. We also need to further investigate the performance of the new method in a restarting mode and the critical role of the temperature in the whole process.

\section{Acknowledgments}

The contributions and assistance provided by Ralph J. Houston is greatly appreciated. We also thank Arjo Wiggins Ltd for providing their paper samples. The partial financial support from the University of Patras, EPSRC and Faperj is gratefully acknowledged. 

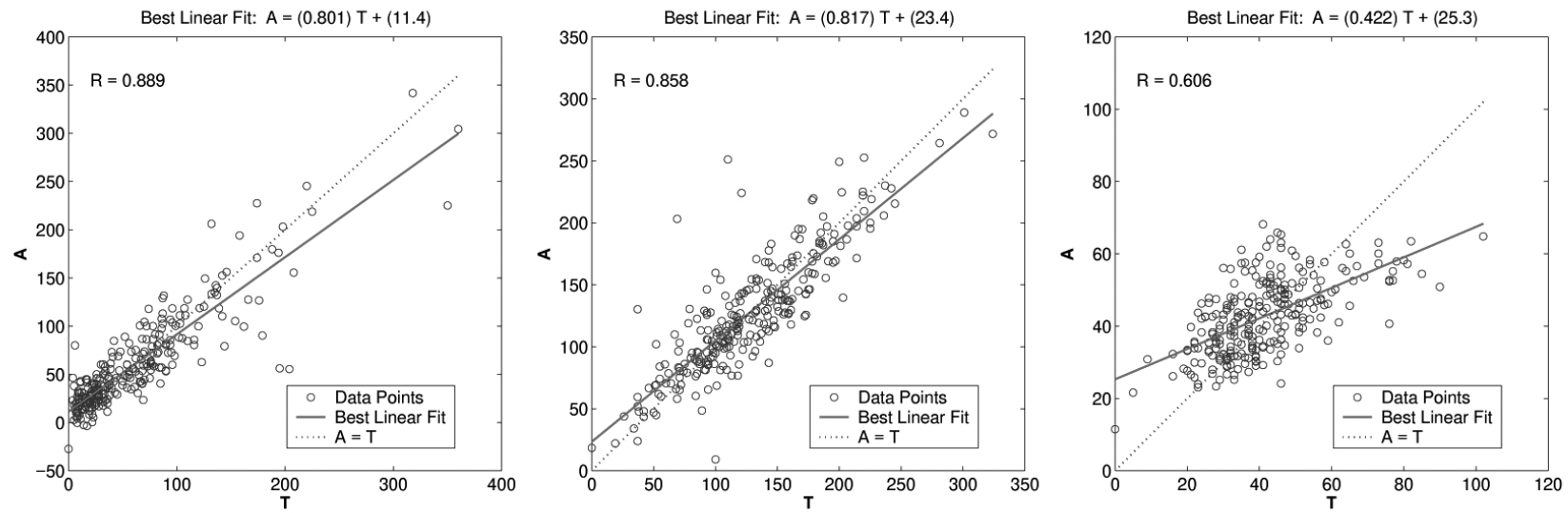

FIG. 3: Linear regression for outputs 1, 2 and 3 of an MLP network with 4 inputs and 27 hidden nodes trained with cholesterol data and the Hybrid Training Scheme. The regression R-values are shown on top left.
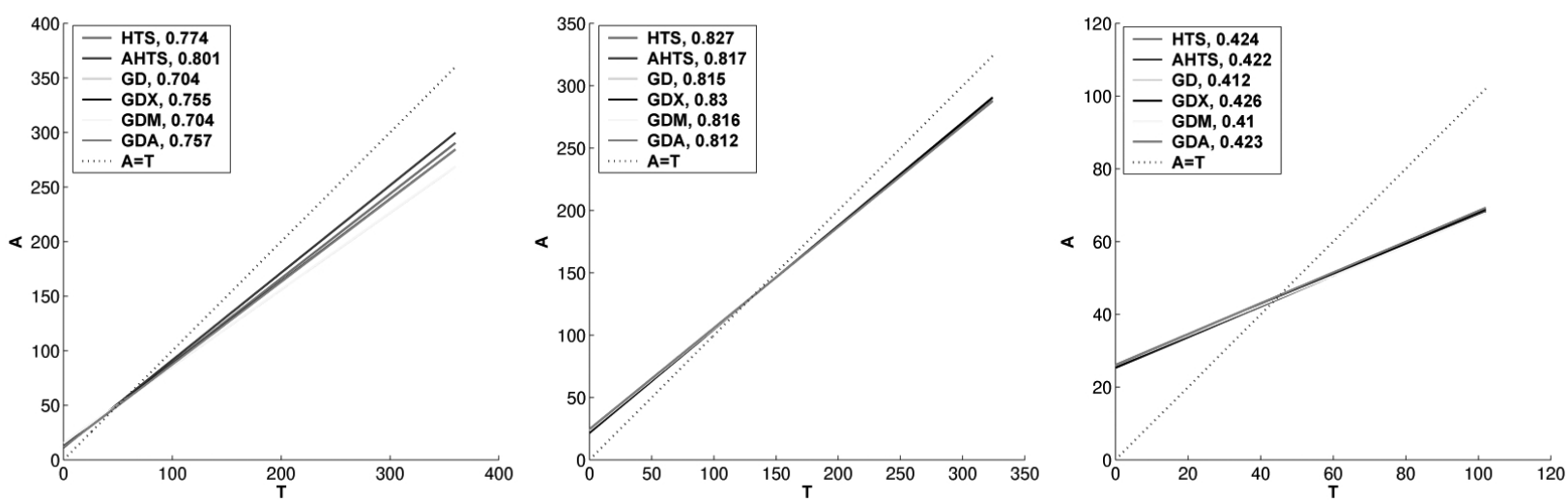

FIG. 4: Linear regression for outputs 1, 2 and 3 of MLP networks with optimal topologies (as shown in Table I) trained with cholesterol data and various learning algorithms. The slope values for each algorithm is shown on top left.

[2] A. D. Anastasiadis and G. D. Magoulas, The European Physical Journal B 50, 277 (2006).

[3] M. Boyukata, Y. Kocyigit, and Z. B. Guvenc, Brazilian Journal of Physics 36, 730 (2006).

[4] L. Dolmatova, C. Ruckebusch, N. Dupuy, J.-P. Huvenne, and P. Legrand, Chemometrics and Intelligent Laboratory Systems 36, 125 (1997).

[5] B. Schölkopf, C. Burges, and A. Smola, Advances in Kernel Methods-Support Vector Learning (MIT Press, Cambridge, MA, 1999).

[6] A. D. Anastasiadis, G. D. Magoulas, and M. N. Vrahatis, Pattern Recognition Letters 26, 1926 (2005).

[7] A. D. Anastasiadis, G. D. Magoulas, and M. N. Vrahatis, Neurocomputing 64,253 (2005).

[8] G. D. Magoulas and M. N. Vrahatis, Neural, Parallel and Scientific Computations 8, 147 (2000).

[9] R. Battiti, Neural Computation 4, 141 (1992).

[10] P. P. Van der Smagt, Neural Networks 7, 1 (1994).

[11] J. Nocedal, Acta Numerica 1, 199 (1992).

[12] G. Gyorgyi, Physics Reports 342, 263 (2001).

[13] C. Tsallis and D. A. Stariolo, Physica A 233, 395 (1996).

[14] C. Tsallis, Statistical Physics 52, 479 (1988).

[15] A. D. Anastasiadis and G. D. Magoulas, Physica A 344, 372 (2004).

[16] C. Tsallis, Brazilian Journal of Physics 29, 1 (1999).
[17] C. Tsallis, Physica D 193, 3 (2004).

[18] H. Shibata, Physica A: Statistical Mechanics and its Applications 317, 391 (2003).

[19] W. H. Siekman, Chaos, Solitons and Fractals 16, 119 (2003).

[20] U. H. E. Hansmanna and Y. Okamotob, Brazilian Journal of Physics 29, 187 (1999).

[21] A. C. Tsallis, C. Tsallis, A. C. N. Magalhaes, and F. A. Tamarit, Complexus 1, 181 (2003).

[22] S. M. D. Queirós, L. G. Moyano, J. de Souza, and C. Tsallis, The European Physical Journal B 55, 161 (2007).

[23] J. E. Straub and I. Andricioaei, Brazilian Journal of Physics 29, 179 (1999).

[24] M. P. de Albuquerque, I. A. Esquef, A. R. G. Mello, and M. P. de Albuquerque, Pattern Recognition Letters 25, 1059 (2004).

[25] M. Gell-Mann and C. Tsallis, Nonextensive EntropyInterdisciplinary Applications (Oxford University Press, 2004).

[26] D. E. Rumelhart, G. E. Hinton, and R. J. Williams, in Parallel Distributed Processing:Explorations in the Microstructure of Cognition 1, edited by D. E. Rumelhart and J. L. McClelland (MIT Press, 1986), pp. 318-362.

[27] C. Tsallis, R. S. Mendes, and A. R. Plastino, Physica A: Statistical Mechanics and its Applications 261, 534 (1998).

[28] D. Kalamatianos, P. Wellstead, J. Edmunds, and P. Liatsis, Review of Scientific Instruments 77 (2006). 
[29] C. Bishop, Neural Networks for Pattern Recognition (Clarendon Press, Oxford, UK, 1995).

[30] N. Purdie, E. A. Lucas, and M. B. Talley, Clinical Chemistry 38, 1645 (1992).
[31] S. G. and W. Cochran, Statistical Methods (Iowa State University Press, 1989). 